\title{
Thalidomide and lenalidomide for recurrent ovarian cancer: A systematic review of the literature
}

\author{
CLEMENS B. TEMPFER ${ }^{1}$, BEATE SCHULTHEIS ${ }^{2}$, ZIAD HILAL ${ }^{1}$, ASKIN DOGAN ${ }^{1}$ and GÜNTHER A. REZNICZEK ${ }^{1}$ \\ Departments of ${ }^{1}$ Obstetrics and Gynecology, and ${ }^{2}$ Hematology and Oncology, \\ Marien Hospital Herne, Ruhr University Bochum, D-44625 Herne, Germany
}

Received February 16, 2017; Accepted June 27, 2017

DOI: $10.3892 / \mathrm{ol} .2017 .6578$

\begin{abstract}
The present review aimed to assess the safety and efficacy of thalidomide and lenalidomide, two immunomodulatory drugs with anti-angiogenic properties, in women with recurrent ovarian, fallopian tube, and primary peritoneal cancer. A systematic review of the literature was conducted whereby Medline and the Cochrane Central Register of Controlled Trials were searched using terms associated with thalidomide, lenalidomide, and recurrent ovarian, fallopian tube and primary peritoneal cancer. Published English language case reports, trials and studies that described the safety and efficacy of thalidomide or lenalidomide alone, or in combination with other drugs were reviewed. A total of 16 clinical studies involving 394 patients treated with thalidomide $(n=188)$, lenalidomide $(n=77)$ and 129 controls were identified, including five case reports $(n=6)$, three case series $(n=45)$, two phase I trials $(n=27)$, four phase II trials $(n=109)$, and two randomized phase III trials $(n=207)$. In a pooled analysis of thalidomide investigated as a single drug, the overall clinical benefit rate was $43 \%$ (43/99) with a mean time to progression of 5.6 months. The response rate (complete response + partial response) was $25 \%$. In a phase III trial, the combination of thalidomide and topotecan significantly increased the overall response rate compared with topotecan alone [14/30 (47\%) vs. 8/39 (21\%)]. In another phase III trial involving women with asymptomatic biochemical recurrence, compared with tamoxifen, thalidomide was not more effective. Lenalidomide was investigated in three phase I trials and in one phase II trial with an overall clinical benefit rate of $52 \%$ $(34 / 65)$, and a mean time to progression of 4.6 months. The response rate (complete response + partial response) was $6 \%$. Systemic toxicity of both drugs was noted in $>77 \%$ of patients with pneumonitis/pneumonia, fatigue, neuropathy and venous
\end{abstract}

Correspondence to: Professor Clemens B. Tempfer, Department of Obstetrics and Gynecology, Marien Hospital Herne, Ruhr University Bochum, Hoelkeskampring 40, D-44625 Herne, Germany E-mail: clemens.tempfer@rub.de

Key words: ovarian cancer, recurrence, anti-angiogenesis, thalidomide, lenalidomide, therapy response, efficacy, safety thromboembolism reported as the most common side effects. Thalidomide and lenalidomide are moderately active in recurrent ovarian cancer. Thalidomide possesses synergistic effects with topotecan. The toxicity of both drugs is considerable and there is a greater amount of data available for thalidomide compared to lenalidomide.

\section{Introduction}

Women with ovarian cancer usually present at an advanced stage with FIGO stage III and IV disease in $>75 \%$ of cases (1). Therefore, the prognosis of ovarian cancer is poor with high recurrence rates between 60 and $85 \%$ within five years despite aggressive multimodality treatment with cytoreductive surgery and adjuvant cytotoxic chemotherapy (2). In order to improve the prognosis of women with ovarian cancer, alternative therapy modalities have been explored, among them a variety of anti-angiogenic drugs such as bevacizumab, pazopanib, and nintedanib (3). Specifically, bevacizumab, a monoclonal humanized antibody against the vascular endothelial growth factor (VEGF), has been shown to significantly prolong recurrence-free and progression-free survival after first-line therapy $(4,5)$ and in women with platinum-sensitive (6) and platinum-resistant recurrence (7). To date, no effect on overall survival was ascertained. Based on the results of randomized clinical trials (4-7), adding bevazicumab to standard systemic chemotherapy has become the standard of care in the adjuvant and recurrent situations.

Another anti-angiogenic agent, pazopanib, an oral, tyrosine kinase inhibitor of the VEGF receptors 1, 2 and 3, the platelet-derived growth factor receptor (PDGFR), and c-Kit, has been tested as a maintenance treatment after initial surgery and adjuvant chemotherapy. In a large randomized trial, maintenance therapy with pazopanib $800 \mathrm{mg}$ once per day or placebo for up to 24 months, significantly prolonged the duration of progression-free survival (PFS) from a median of 12.3 to 17.9 months. Overall survival was not improved (8). Besides bevacizumab and pazopanib, a third anti-angiogenic drug, nintedanib, has been demonstrated to improve PFS in ovarian cancer patients. Nintedanib is an oral triple angiokinase inhibitor of the VEGF- and PDGF-receptors and the fibroblast growth factor receptor (FGFR). In a randomized phase III trial, maintenance treatment with $200 \mathrm{mg}$ of nintedanib twice daily on days 2 to 21 of every 3 week cycle for up to 120 weeks was 
tested against placebo. Median PFS was significantly longer in the nintedanib group, although the absolute difference was small (17.2 vs. 16.6 months) (9). Taken together, these trials demonstrate that blocking tumor angiogenesis at various levels is a successful strategy to delay disease progression in ovarian cancer. Therefore, it is reasonable to investigate additional anti-angiogenic drugs in this patient population.

Thalidomide and lenalidomide are such drugs, because they are immunomodulatory agents with strong anti-angiogenic properties. Thalidomide inhibits the processing of mRNA encoding different peptide molecules such as tumor necrosis factor- $\alpha$ (TNF- $\alpha$ ) and VEGF (10). In addition, thalidomide modulates intracellular signaling pathways via the mediation of VEGF, phosphoinositide-kinase/protein kinase B and nuclear factor $-\kappa \mathrm{B}(\mathrm{NF}-\kappa \mathrm{B})$, and the mammalian target of rapamycin (mTOR), which integrates these signaling systems (11). In pre-clinical studies, thalidomide has been shown to suppress malignant cell proliferation and angiogenesis as well as invasion and metastasis (12).

Historically, thalidomide was developed as a drug for the treatment of morning sickness in pregnant women (13). After its teratogenic effects leading to limb defects became apparent in the early sixties of the 20th century (14), thalidomide was retracted worldwide from the market. However, the drug has made a remarkable comeback in the last decade as a highly effective agent for leprosy, multiple myeloma, and Crohn's disease (15).

For example, thalidomide is being used effectively in the treatment of inflammatory bowel diseases refractory to first-line and second-line treatments. In a recent systematic literature review, Bramuzzo et al identified 2 randomized controlled trials and 29 uncontrolled trials with a total of 489 patients (16). Thalidomide induced a clinical response in 296/427 (69\%) patients. Clinical remission was achieved in 220/427 (51\%) cases. Maintenance of remission was reported in 128/160 (80\%) patients at 6 months and in 96/133 (72\%) at 12 months. Reduction in steroid dosage, clinical improvement of fistulas, and endoscopic improvement were also reported. Neurological disturbances in 341/530 (64\%) patients accounted for most adverse events and were the most frequent cause of drug withdrawal.

Regarding the use of thalidomide as an antitumor drug, a number of malignant diseases have been investigated, among them glioblastoma (17), hepatocellular carcinoma (18), and multiple myeloma (19). For example, in a meta-analysis of randomized trials, Gao et al summarized the results of 22 randomized trials with 9,098 multiple myeloma patients (19). Induction therapy with thalidomide significantly improved the overall response rate, progression-free survival, and overall survival in patients who were not allowed to receive autologous stem cell transplantation. Among patients who were allowed to receive autologous stem cell transplantation, induction treatment with thalidomide significantly improved the overall response rate and progression-free survival, but not overall survival. The most notable side effect of thalidomide in these trials was an increased rate of venous thromboembolism (VTE).

Lenalidomide has immunomodulatory and anti-angiogenic effects. For example, it modulates the substrate specificity of the CRL4 (CRBN) E3 ubiquitin ligase complex (20). Polyubiquitination and subsequent proteasomal degradation of IKZF1 and IKZF3 in multiple myeloma and CK1 $\alpha$ in del (5q) myelodysplastic syndrome has been described as the basis of the therapeutic efficacy of lenalidomide. Harnessing ubiquitin ligase substrate specificity facilitates the degradation of other 'undruggable' proteins and thus allows for the separation of detrimental side effects of IMiD compounds from those associated with therapeutic efficacy $(20,21)$. Lenalidomide $\left(\right.$ Revlimid $^{\circledR}$ ) has been approved by the FDA for previously treated multiple myeloma in combination with dexamethasone.

Based on the well-known fact that anti-angiogenesis is effectively delaying disease progression in ovarian cancer patients, the compassionate use of thalidomide has been reported in heavily pre-treated patients with ovarian cancer recurrence (22-24). These case reports indicated that thalidomide may be active in ovarian cancer. In order to further highlight the safety and efficacy of thalidomide and lenalidomide in patients with recurrent ovarian, fallopian tube, and primary peritoneal cancer, we performed a systematic literature review of retrospective and prospective clinical trials evaluating the efficacy and safety of these two anti-angiogenic agents.

\section{Materials and methods}

A systematic review of the literature was conducted to identify studies with thalidomide and lenalidomide in women with recurrent ovarian, fallopian tube, or primary peritoneal cancer. The databases Medline and the Cochrane Central Register of Controlled Trials were searched for published English language case reports, clinical trials and other studies that described the safety and efficacy of thalidomide or lenalidomide alone or in combination with other drugs, using the search terms 'thalidomide' [MeSH Terms] OR 'thalidomide' [All Fields]) AND ('ovarian neoplasms' [MeSH Terms] OR ('ovarian' [All Fields] AND 'neoplasms' [All Fields] ) OR 'ovarian neoplasms' [All Fields] OR ('ovarian' [All Fields] AND 'cancer' [All Fields]) OR 'ovarian cancer' [All Fields]). In a second step, we used the search terms 'lenalidomide' [Supplementary Concept] OR 'lenalidomide' [All Fields]) AND ('ovarian neoplasms' [MeSH Terms] OR ('ovarian' [All Fields] AND ‘neoplasms' [All Fields]) OR 'ovarian neoplasms' [All Fields] OR ('ovarian' [All Fields] AND 'cancer' [All Fields]) OR 'ovarian cancer' [All Fields]).

Abstracts were screened for the following eligibility criteria: Studies describing at least one woman with recurrent ovarian, fallopian tube or primary peritoneal cancer treated with thalidomide or lenalidomide with description of the safety and/or efficacy and/or side effects of this treatment. Duplicate publications were excluded. No size or design restrictions were applied, ie case reports, case series, retrospective and prospective cohort studies, case control studies, and randomized controlled trials were allowed. Relevant full text articles were retrieved and analyzed for outcomes related to the goal of this review-to assess the safety, efficacy, and side effects of thalidomide and lenalidomide in women with recurrent ovarian, fallopian tube or primary peritoneal cancer.

\section{Results}

In a systematic literature search of the databases PubMed and Cochrane Central Register of Controlled Trials (search 


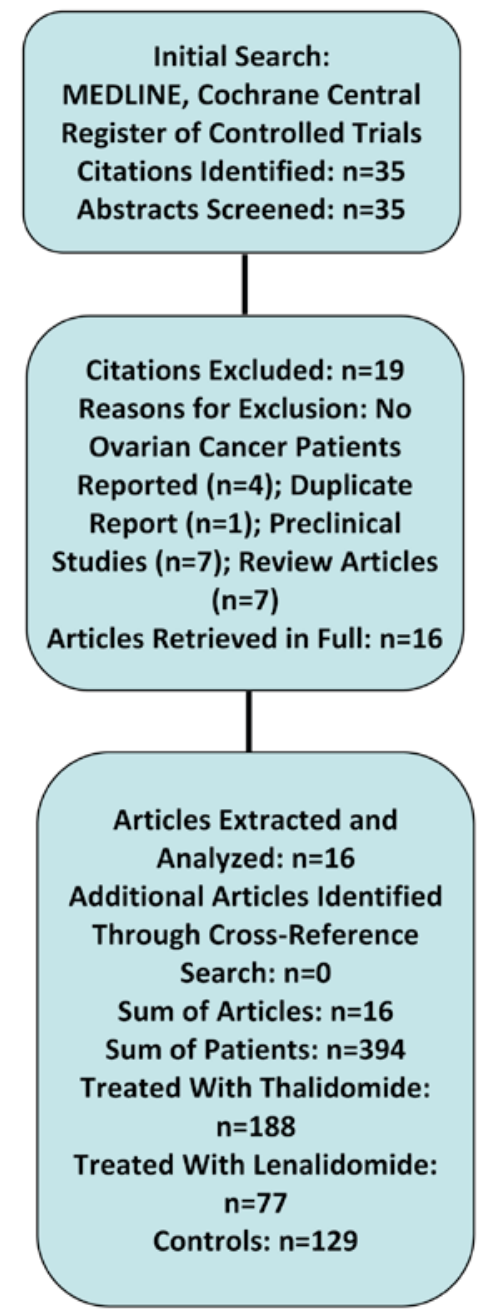

Figure 1. Flow diagram of the literature search algorithm.

date 01-11-2016) using the search terms 'thalidomide' [MeSH Terms] OR 'thalidomide' [All Fields]) AND ('ovarian neoplasms' [MeSH Terms] OR ('ovarian' [All Fields] AND 'neoplasms' [All Fields]) OR 'ovarian neoplasms' [All Fields] OR ('ovarian' [All Fields] AND 'cancer' [All Fields]) OR 'ovarian cancer' [All Fields]), we identified 32 studies $(15,22-52)$. In a second step, we used the search terms 'lenalidomide' [Supplementary Concept] OR 'lenalidomide' [All Fields]) AND ('ovarian neoplasms' [MeSH Terms] OR ('ovarian' [All Fields] AND 'neoplasms' [All Fields]) OR 'ovarian neoplasms' [All Fields] OR ('ovarian' [All Fields] AND ‘cancer' [All Fields]) OR 'ovarian cancer' [All Fields]) and identified 3 additional studies (53-55). After screening all abstracts of these studies, we excluded 4 studies (51,53-55) because they did not report on patients with ovarian cancer and one study (52) because the patients reported in this study were already published in another trial by the same authors (42). Further 7 citations comprised preclinical studies describing in vitro and in vivo data on the antitumor effects of thalidomide and lenalidomide (25-31). Seven citations were review articles not containing individual patients data (15,32-37). Thus, in summary, we included 16 clinical studies describing 394 patients treated with thalidomide $(n=188)$, lenalidomide $(\mathrm{n}=77)$, and 129 controls. These studies included 5 case reports $(n=6), 3$ case series $(n=45), 2$ phase I trials $(n=27), 4$ phase II trials $(n=109)$, and 2 randomized phase III trials $(n=207)$. Table I shows the study characteristics and results of the 5 case reports and 11 clinical trials in detail. Figure 1 shows a flow diagram of the literature search algorithm. In a pooled analysis of trials with thalidomide tested as a single drug, the overall response rate was $43 \%$ (43/99) with a mean time to progression of 5.6 months. Among these patients, complete response was observed in $11 \%$, partial response in $13 \%$, and stable disease in $19 \%$. The dosage of thalidomide used in these studies ranged from 100 to $1,200 \mathrm{mg} /$ day. In one phase III trial, the combination of thalidomide and topotecan significantly increased the overall response rate compared to topotecan alone (14/30 [47\%] vs. 8/39 [21\%]). In another phase III trial of women with biochemical ovarian cancer recurrence, thalidomide was not more effective than tamoxifen.

Lenalidomide was investigated in 3 phase I trials and in one phase II trial with an overall response rate of 52\% (34/65) and a mean time to progression of 4.6 months. Among these patients, complete response was observed in $0 \%$, partial response in $6 \%$, and stable disease in $46 \%$. The dosage of lenalidomide used in these studies ranged from 5 to $25 \mathrm{mg} /$ day.

Systemic toxicity of both drugs was noted in up to $77 \%$ of patients with pneumonitis/pneumonia, fatigue, neutropenia, neuropathy, and VTE cited as the most common side effects. Table II shows the side effects of thalidomide observed in 12 clinical trials with 317 patients in detail. Grade 3 or 4 adverse events were noted in 61/132 (46\%) of women with thalidomide as a single treatment. Specifically, the most often cited side effects of thalidomide were pulmonary problems/pneumonia/pneumonitis (4 studies), neuropathy (4 studies), VTE (3 studies), weakness/fatigue (3 studies), constipation (3 studies), and somnolence (3 studies). Table III shows the side effects of lenalidomide in 4 clinical trials with 77 patients in detail. 43/65 (66\%) patients with lenalidomide as a single treatment showed grade 3 adverse events and 7/65 (11\%) patients showed grade 4 adverse events. The most often cited side effects of lenalidomide were neutropenia (4 studies), VTE (2 studies), rash (2 studies), constipation (2 studies), and weakness (2 studies).

Experimental studies. Several experimental studies assessed the antitumor effects of thalidomide in ovarian cancer cell lines, ex vivo, and in vivo models. For example, Kobayashi et al examined whether thalidomide is able to suppress the expression of urokinase-type plasminogen activator receptor (uPAR) mRNA and protein in the human ovarian cancer cell line HRA (28). They found that thalidomide had multiple synergistic effects. Specifically, it suppressed the expression of UPAR mRNA and protein as well as the $\mathrm{NF}-\kappa \mathrm{B}$ activation system, which is necessary for the transforming growth factor (TGF)- $\beta 1$-induced increase in uPAR expression. Furthermore, the once-daily intraperitoneal administration of thalidomide (400 $\mu \mathrm{g} / \mathrm{g}$ body weight/day) decreased progressive growth of HRA tumors and ascites formation in an in vivo animal model. The once-daily intraperitoneal administration of thalidomide in combination with paclitaxel significantly decreased the growth of HRA cells in a synergistic fashion. These data suggest that thalidomide downregulates constitutive and TGF- $\beta 1$-stimulated UPAR mRNA and protein expression via the suppression of $\mathrm{NF}-\kappa \mathrm{B}$ and may have synergistic effects 


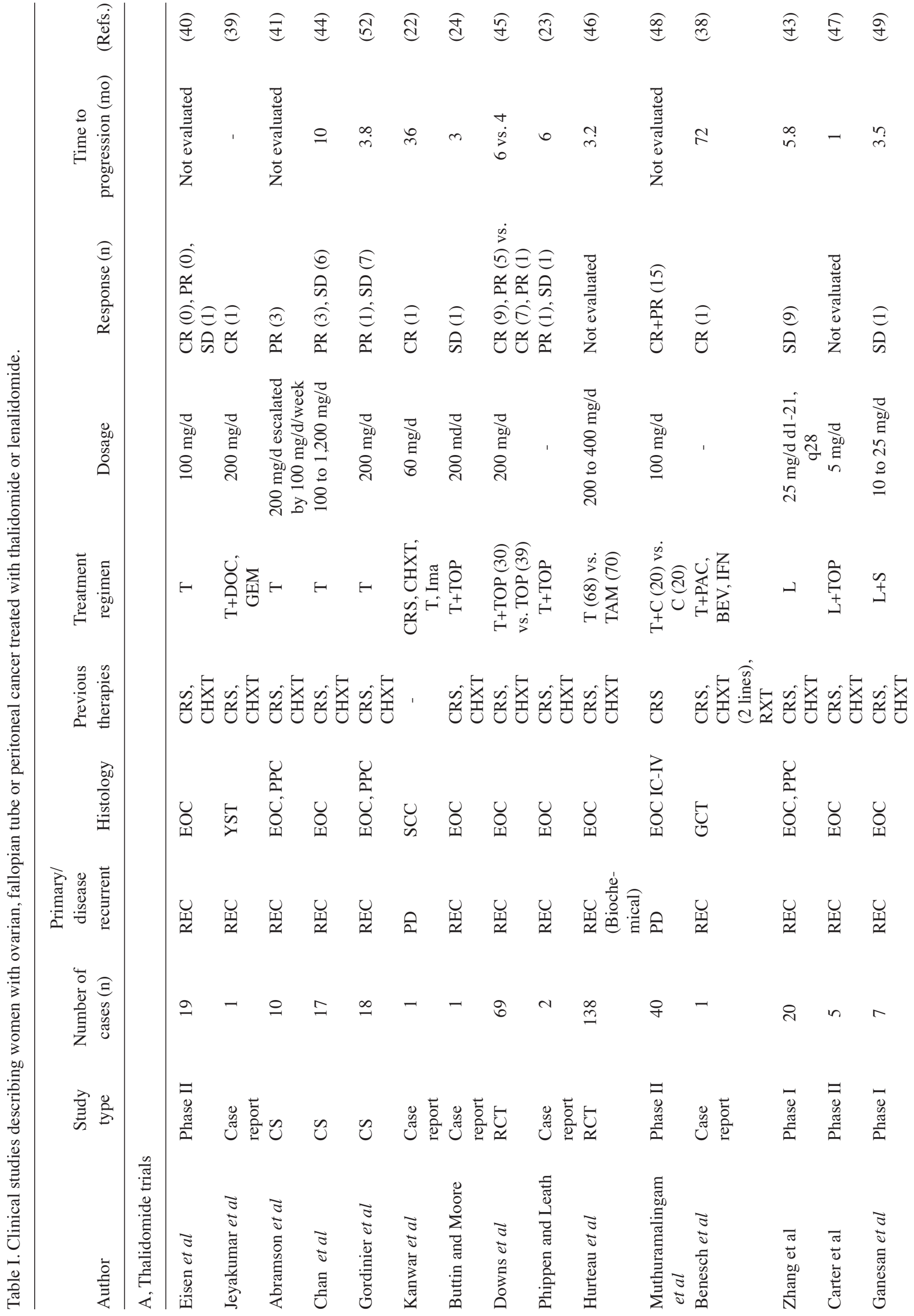




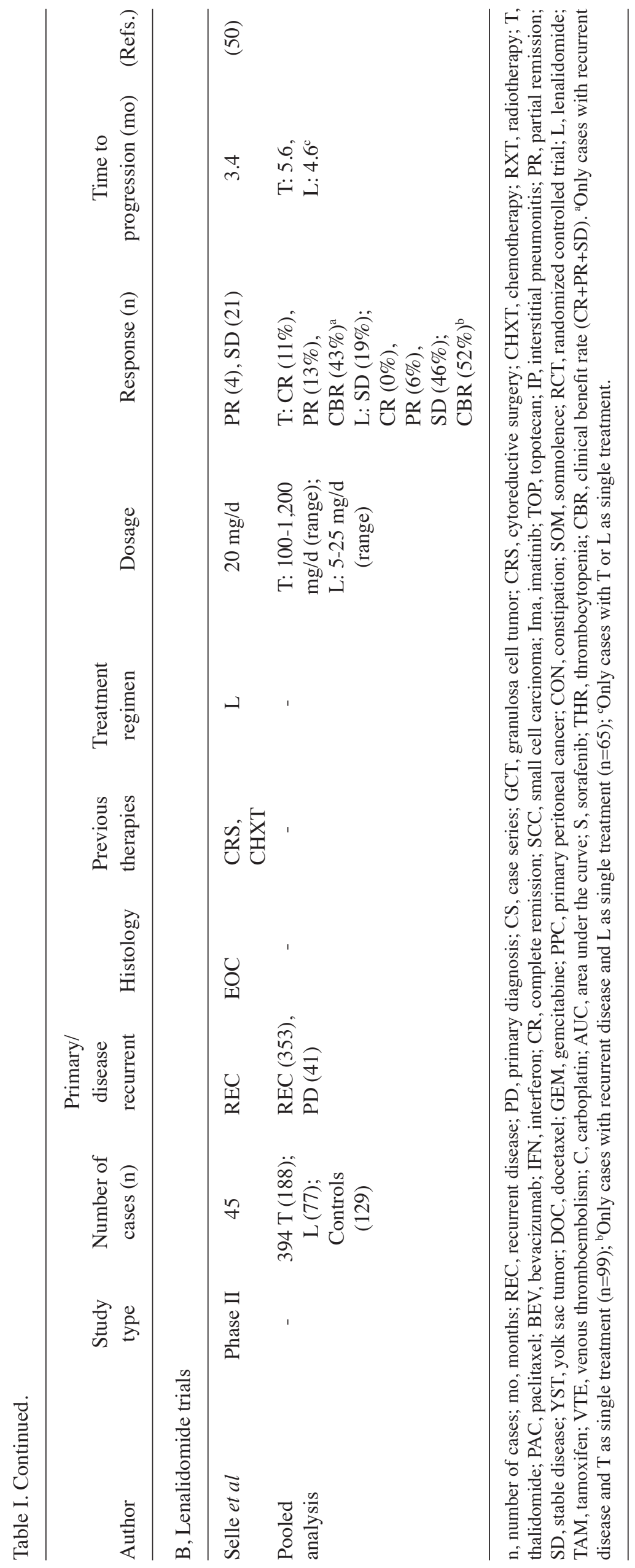


Table II. Clinical studies describing the toxicity of thalidomide in women with ovarian, fallopian tube or peritoneal cancer.

\begin{tabular}{|c|c|c|c|c|c|}
\hline Author & $\begin{array}{l}\text { Number of } \\
\text { cases }(n)\end{array}$ & $\begin{array}{l}\text { Treatment } \\
\text { regimen }\end{array}$ & $\begin{array}{l}\text { Grade } 3 \\
\text { events }\end{array}$ & $\begin{array}{l}\text { Grade } 4 \\
\text { events }\end{array}$ & (Refs.) \\
\hline Eisen et al & 19 & $\mathrm{~T}$ & 0 & 0 & $(40)$ \\
\hline Jeyakumar et al & 1 & $\begin{array}{c}\text { T+DOC } \\
\text { GEM }\end{array}$ & 0 & $\begin{array}{c}1 \text { (Bowel } \\
\text { otbstruction) }\end{array}$ & (39) \\
\hline Abramson et al & 10 & $\mathrm{~T}$ & 0 & 0 & $(41)$ \\
\hline Chan et al & 17 & $\mathrm{~T}$ & $\begin{array}{c}2 \text { (VTE), } 1(\mathrm{CON}), \\
1 \text { (Confusion), } \\
1 \text { (Speech impairment) }\end{array}$ & & (44) \\
\hline Gordinier et al & 18 & $\mathrm{~T}$ & $\begin{array}{c}8 \text { (Dyspnea), } 2(\mathrm{CON}) \\
2 \text { (Sedation) }\end{array}$ & 0 & $(42)$ \\
\hline Kanwar et al & 1 & $\begin{array}{l}\text { CRS, CHXT, } \\
\text { T, IMA }\end{array}$ & 3 (Neurological) & 0 & $(22)$ \\
\hline Buttin and Moore & 1 & $\mathrm{~T}+\mathrm{TOP}$ & $1(\mathrm{IP})$ & 0 & $(24)$ \\
\hline Downs et al & 69 & $\begin{array}{l}\text { T+TOP (30) } \\
\text { vs. TOP (39) }\end{array}$ & $\begin{array}{c}28 \text { (Neutropenia), } \\
6 \text { (Thrombocytopenia), } \\
4 \text { (Anemia), } 4 \text { (Neurologic), } \\
3 \text { Pulmonary, } 2 \text { (Infection), } \\
2 \text { (Constitutional), } 1 \text { (VTE), } \\
1 \text { (Dermatology), } 1 \text { (Ocular), } \\
1 \text { (Gastrointestinal) }\end{array}$ & & $(45)$ \\
\hline Phippen and Leath & 2 & $\mathrm{~T}+\mathrm{TOP}$ & 2 (Fatigue) & 0 & $(23)$ \\
\hline Hurteau et al & 138 & $\begin{array}{l}\text { T (68) vs. } \\
\text { TAM (70) }\end{array}$ & $\begin{array}{c}8 \text { (Constitutional), } 7 \text { (Somnolence), } \\
7 \text { (Neurologic), } 6 \text { (Pulmonary), } \\
5 \text { (Dermatologic), } 3 \text { (Pain), } \\
3 \text { (Gastrointestinal), 2 (VTE) }\end{array}$ & $\begin{array}{c}2(\mathrm{VTE}), \\
1 \text { (Somnolence) }\end{array}$ & $(46)$ \\
\hline $\begin{array}{l}\text { Muthuramalingam } \\
\text { et al }\end{array}$ & 40 & $\begin{array}{r}\mathrm{T}+\mathrm{C}(20) \\
\text { vs. C (20) }\end{array}$ & - & - & $(48)$ \\
\hline Benesch et al & 1 & $\begin{array}{l}\text { T+PAC, } \\
\text { BEV, IFN }\end{array}$ & 0 & 0 & $(38)$ \\
\hline $\begin{array}{l}\text { Pooled } \\
\text { analysis }\end{array}$ & $\begin{array}{c}317 \text { T (188); } \\
\text { Controls (129) }\end{array}$ & - & \multicolumn{3}{|c|}{$61 / 132(46 \%)^{\mathrm{a}}$} \\
\hline
\end{tabular}

n, number of cases; T, thalidomide; DOC, docetaxel; GEM, gemcitabine; VTE, venous thromboembolism; CON, constipation; CRS, cytoreductive surgery; CHXT, chemotherapy; IMA, imatinib; TOP, topotecan; IP, interstitial pneumonitis; C, carboplatin; PAC, paclitaxel; BEV, bevacizumab; IFN, interferon. ${ }^{a}$ Only cases with $\mathrm{T}$ as single treatment $(\mathrm{n}=132)$.

with paclitaxel. Piura et al assessed the effect of thalidomide on the ovarian cancer cell secretome of SKOV-3 cells (31). TNF- $\alpha$, interleukin (IL)-6 and matrix metalloproteinase (MMP) secretion in epithelial ovarian cancer cells. In these experiments, thalidomide significantly decreased the secretion of TNF- $\alpha$, MMP-9, and MMP-2.

Bauer et al evaluated the antiangiogenic activity of interferon (IFN)- $\alpha 2 b$ and thalidomide. They used a murine dermis model in nude mice. The combination of IFN- $\alpha 2 b$ and thalidomide had synergistic effects in reducing angiogenesis and tumor activity in this model (27). In another murine xenograft model, Li et al studied the effect of thalidomide alone or in combination with cytoxan on the growth and angiogenesis of human ovarian cancer cells transplanted subcutaneously into nude mice (29). Both thalidomide and the combination of thalidomide and cytoxan reduced the expression of VEGF mRNA and serum levels, tumor volume, microvessel density, and macroscopic tumor volumes.
Taken together, the experimental evidence strongly suggests that thalidomide is active in ovarian cancer through the downregulation of constitutive and TGF-beta1-stimulated uPAR expression via the suppression of NF- $\mathrm{NB}$. In addition, it affects the ovarian cancer cell secretome and reduces angiogenesis, proliferation, and tumor growth.

Thalidomide trials. In the largest trial, sponsored by the Gynecologic Oncology Group (GOG), Hurteau et al analyzed 138 women with ovarian, fallopian tube or peritoneal cancer FIGO stages III and IV who were free of disease following first-line chemotherapy and subsequently developed biochemical recurrence (46). Biochemical recurrence was defined as a rising CA-125 exceeding twice the upper limit of normal without evidence of disease in imaging studies. Women were randomized to oral thalidomide $200 \mathrm{mg}$ daily with escalation to a maximum of $400 \mathrm{mg}$ or tamoxifen $20 \mathrm{mg}$ orally twice daily for up to 1 year. Treatment was given until progression or limiting 
Table III. Clinical studies describing the toxicity of lenalidomide in women with ovarian, fallopian tube or peritoneal cancer.

\begin{tabular}{|c|c|c|c|c|c|}
\hline Author & $\begin{array}{l}\text { Number of } \\
\text { cases }(n)\end{array}$ & $\begin{array}{l}\text { Treatment } \\
\text { regimen }\end{array}$ & Grade 3 events & Grade 4 events & (Refs.) \\
\hline Zhang et al & 20 & $\mathrm{~L}$ & $\begin{array}{l}7 \text { (NEU), } 2 \text { (Anemia), } 1 \text { (Fatigue), } \\
1 \text { (Nausea), } 1 \text { (Diarrhea), } 1 \text { (VTE) }\end{array}$ & 0 & $(43)$ \\
\hline Carter et al & 5 & $\mathrm{~L}+\mathrm{TOP}$ & $\begin{array}{l}3 \text { (NEU), } 2 \text { (THR), } 2 \text { (Anemia), } \\
1 \text { (Fatigue), } 1 \text { (VTE) }\end{array}$ & 0 & $(47)$ \\
\hline Ganesan et al & 7 & $\mathrm{~L}+\mathrm{S}$ & $\begin{array}{l}7 \text { (NEU), } 2 \text { (THR) } \\
2 \text { (Rash), } 1 \text { (VTE) }\end{array}$ & $1(\mathrm{PE})$ & $(49)$ \\
\hline Selle et al & 45 & $\mathrm{~L}$ & $\begin{array}{l}10 \text { (NEU), } 3 \text { (Pain), } 3 \text { (Obstruction), } \\
2 \text { (VTE), } 2 \text { (Edema), } 2 \text { (Vomiting), } \\
1 \text { (Dyspnea), } 1 \text { (Diarrhea) }\end{array}$ & $\begin{array}{l}3 \text { (Neutropenia), } \\
3 \text { (VTE) }\end{array}$ & $(50)$ \\
\hline Pooled analysis & $\begin{array}{l}77 \text { L (77); } \\
\text { Controls (0) }\end{array}$ & - & $43 / 65(66 \%)^{\mathrm{a}}$ & $7 / 65(11 \%)^{\mathrm{a}}$ & \\
\hline
\end{tabular}

$\mathrm{n}$, number of cases; L, lenalidomide; NEU, neutropenia; VTE, venous thromboembolism; TOP, topotecan; THR, thrombocytopenia; S,

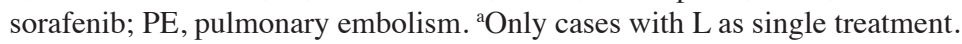

toxicity. In this trial, thalidomide did not reduce the recurrence rate relative to tamoxifen. Specifically, thalidomide vs. tamoxifen was associated with a similar risk of progression [hazard ratio $(\mathrm{HR})=1.31,95 \%$ confidence interval $(\mathrm{CI})=0.93-1.85]$. Of note, thalidomide even resulted in a significantly increased risk of death $(\mathrm{HR}=1.76,95 \% \mathrm{CI}=1.16-2.68)$ and had significantly more grade 3 and 4 toxicities (55\% vs. 3\%). The most common grade 3 and 4 toxicities of thalidomide were constitutional (12\%), somnolence (12\%), pulmonary (9\%), VTE $(6 \%)$ and peripheral neurologic toxicity $(6 \%)$. This trial demonstrates that thalidomide should not be used in women with biochemical recurrence in order to prolong time to progression. In this indication, thalidomide clearly does more harm than good with significant increases in morbidity and even mortality.

In contrast to the Hurteau et al (46) trial, the second largest trial was positive. In a randomized phase III trial, Downs et al and compared the response rates of 69 women with recurrent ovarian cancer treated with topotecan or topotecan and thalidomide (45). Eligible patients had recurrent epithelial ovarian carcinoma with measurable disease or elevated CA 125 values. Patients received topotecan at a dose of $1.25 \mathrm{mg} / \mathrm{m}^{2}$ on days 1 through 5 of a 21-day cycle or topotecan and thalidomide with a starting dose of $200 \mathrm{mg}$ per day and then increasing the dose as tolerated. In this positive trial, the overall response rate in the topotecan only arm was $21 \%$ [complete responses (CR) in $18 \%$ and partial responses (PR) in $3 \%$ of patients] compared with $47 \%$ in the topotecan and thalidomide arm (CR 30\%, PR 17\%). This difference was clinically and statistically significant $(\mathrm{P}=0.03)$. The median progression-free survival was significantly longer in the topotecan and thalidomide arm (6 vs. 4 months; $\mathrm{P}=0.02)$. Of note, overall survival time was also longer with topotecan and thalidomide (19 vs. 15 months), but this difference was not statistically significant. In contrast to the Hurteau et al (46) trial, toxicities in this study were similar in both treatment arms. Specifically, there were not more grade 3 and 4 events in the combination arm. This trial is notable due to its randomized design and the positive result indicating a statistically and clinically significant additive antitumor effect of thalidomide in combination with topotecan. Moreover, thalidomide did not increase overall toxicity.

In a prospective phase II trial, Eisen et al found no objective tumor responses in 19 women with recurrent ovarian cancer treated with low dose oral thalidomide (100 mg per day) (40). They reported no toxicity for thalidomide. To the contrary, thalidomide improved appetite and sleeping patterns. In three retrospective case series of 10,17 , and 18 women, Abramson et al (41), Chan et al (44), and Gordinier et al (52) reported response rates of $33 \%$ (3/10), 53\% (9/17), and $44 \%(8 / 18)$, respectively. In all three case series, no complete response was noted. Responses consisted of partial response and stable disease. Toxicity of thalidomide was moderate described as mild weakness, agitation, somnolence, rash, and constipation in one study (41). However, Gordinier et al observed grade 3 dyspnea in 8/18 (44\%) and grade 3 constipation in 2/18 (11\%) patients, respectively (52). In Chan's study, two women experienced grade 3/4 toxicity (constipation and neurologic toxicity) and stopped treatment (44).

Five case reports describing the effects of thalidomide in women with recurrent ovarian cancer were identified. Complete responses under thalidomide were reported in 3 case reports $(22,38,39)$. However, in all 3 cases thalidomide was combined with systemic chemotherapy. In 2 further case reports of 3 patients $(23,24)$, thalidomide combined with systemic chemotherapy achieved a partial response in one and stable disease in 2 patients. In these case reports, thalidomide was combined with topotecan, docetaxel, gemcitabine, and paclitaxel. As relevant toxicity, one case of interstitial pneumonitis was reported among these 6 patients.

Lenalidomide trials. In a prospective phase II trial of 45 women with recurrent ovarian cancer, continuous oral lenalidomide at a dose of $20 \mathrm{mg}$ per day achieved 4 partial responses and 21 cases of stable disease for an overall response rate of $56 \%(25 / 45)(50)$. The most frequent toxicity was hematologic, 
notably grade $3 / 4$ neutropenia in $29 \%$ of patients, along with fatigue $(69 \%)$, gastrointestinal toxicity (constipation $53 \%$, abdominal pain $49 \%$, diarrhea $38 \%$, nausea/vomiting $36 \%$ ) and thrombosis (11\%). Eight patients withdrew due to intolerable toxicity. Thus, this study indicates that lenalidomide as a single treatment has significant activity in this patient population despite a considerable toxicity. In another small phase I/II dose escalation trial, lenalidomide was escalated from 5 to $25 \mathrm{mg}$ per day in $5 \mathrm{mg}$ increments and combined with intravenous topotecan $1.25 \mathrm{mg} / \mathrm{m}^{2}$ on days 1 to 5 of a 21-day cycle (47). Of note, 4 patients discontinued because of dose-limiting toxicity, most commonly grade 4 neutropenia and the study was terminated early for reasons of toxicity.

Lenalidomide was further investigated in 2 phase I trials with 20 and 7 participants $(43,49)$. Nine of 20 women $(45 \%)$ were noted to have stable disease in one trial (43) and 1/7 women $(14 \%)$ in the second trial, in which lenalidomide was combined with sorafenib (49). The toxicities observed were considerable. In the Ganesan et al (49) trial with 7 participants, 15 grade $3 / 4$ events were observed, most notably neutropenia $(n=7)$, thrombocytopenia, rash, VTE, and pulmonary embolism (all $\mathrm{n}=2$ ). In the Zhang et al (43) trial of 20 women, lenalidomide led to 14 grade $3 / 4$ events (neutropenia, $n=7$; anemia, $n=2$; fatigue, nausea, diarrhea, and VTE.

\section{Discussion}

In a systematic literature search, we identified 16 clinical studies with 394 patients treated with thalidomide or lenalidomide. Overall, thalidomide achieved an overall response rate of $43 \%$ with a mean time to progression of 5.6 months and lenalidomide achieved an overall response rate of $52 \%$ with a mean time to progression of 4.6 months. Systemic toxicity of both drugs was considerable and was documented in up to $77 \%$ of patients with pneumonitis/pneumonia, neutropenia, rash, constipation, somnolence, fatigue, neuropathy, and VTE cited as the most common side effects. Based on these data, we conclude that both thalidomide and lenalidomide are active drugs in recurrent ovarian cancer. The toxicity of both drugs, however, is high with pneumonitis/pneumonia, fatigue, neuropathy, neutropenia, and VTE being the most important side effects. More data are available for thalidomide than for lenalidomide. The high toxicity of thalidomide and lenalidomide clearly limits the clinical usefulness of these drugs in the palliative situation of recurrent ovarian cancer.

Thalidomide has synergistic effects with topotecan. This is the notable result of a randomized trial comparing topotecan alone and topotecan combined with thalidomide (45) in women with recurrent ovarian cancer. The combination treatment arm doubled the response rate from 21 to $47 \%$. Moreover, adding thalidomide to the standard topotecan chemotherapy regime did not increase tocixity. Unfortunately, there is no independent confirmation of this result and the number of patients recruited for this trial was low with only 69 probands. Adding the antiangiogenic agent thalidomide to cytotoxic chemotherapy is a reasonable strategy given that other antiangiogenic agents such as bevacizumab have been clearly demonstrated to enhance the antitumoral effect of cytotoxic chemotherapy (3-7). If this additive effect of thalidomide to systemic chemotherapy is confirmed in more trials, adding thalidomide to standard palliative chemotherapy could be a promising strategy to integrate thalidomide into clinical practice.

Both thalidomide and lenalidomide have considerable side effects. For example, grade 3 or 4 adverse events were noted in $61 / 132(46 \%)$ of women with thalidomide as a single treatment. The frequency of severe side effects was even higher with lenalidomide. In 54/65 (77\%) of women treated with lenalidomide as a single treatment, a grade 3 or 4 adverse event was noted. Proteinuria and hypertension, which are well-known side effects of the antiangiogenic agent bevacizumab, have not been noted in women treated with thalidomide or lenalidomide. Thus, the side effect profile of thalidomide and lenalidomide is specific and clearly differs from other antiangiogenic drugs. Typical side effects noted in many studies with thalidomide and lenalidomide are an increased pulmonary tocixity and a high rate of VTE. Thus, prophylactic heparinization may be discussed with patients prior to initiating thalidomide or lenalidomide, especially in those patients with additional VTE risk factors. In view of the fact that all patients with recurrent ovarian, fallopian tube, and peritoneal cancer are in a palliative situation, the pronounced side effect profile of thalidomide and lenalidomide is a severe limitation of these agents. When considering to use these agents, a detailed discussion with the patient about the treatment benefit and the drug-associated toxicity is necessary.

Based on the overall estimation of a response rate of $25 \%$ (complete responses and partial responses) for thalidomide and 6\% (complete responses and partial responses) for lenalidomide, both drugs should not be used routinely in women with recurrent ovarian cancer. In addition, neither thalidomide nor lenalidomide are approved for this patient population. Thus, the use of both drugs in ovarian cancer patients is off-label. This fact may also limit the practical use of thalidomide and lenalidomide in women with recurrent ovarian, fallopian tube, and peritoneal cancer, because off-label use has a number of practical consequences for the treating physician regarding liability, informed consent, and reimbursement issues (54).

In summary, we found that the antiangiogenic agents thalidomide and lenalidomide are active drugs in recurrent ovarian cancer. Thalidomide has synergistic effects with topotecan. The toxicity of both drugs is considerable with pneumonitis/pneumonia, fatigue, neuropathy, and VTE. More data are available for thalidomide than for lenalidomide. Both thalidomide and lenalidomide are not recommended for women with recurrent ovarian cancer. However, the available data support further investigations of the combination of thalidomide and topotecan within the setting of clinical trials.

\section{References}

1. Malvezzi M, Carioli G, Rodriguez T, Negri E and La Vecchia C: Global trends and predictions in ovarian cancer mortality. Ann Oncol 27: 2017-2025, 2016.

2. Foley OW, Rauh-Hain JA and del Carmen MG: Recurrent epithelial ovarian cancer: An update on treatment. Oncology (Williston Park) 27: 288-294, 298, 2003.

3. Wu YS, Shui L, Shen D and Chen X: Bevacizumab combined with chemotherapy for ovarian cancer: An updated systematic review and meta-analysis of randomized controlled trials. Oncotarget 8: 10703-10713, 2017. 
4. Oza AM, Cook AD, Pfisterer J, Embleton A, Ledermann JA, Pujade-Lauraine E, Kristensen G, Carey MS, Beale P, Cervantes A, et al: Standard chemotherapy with or without bevacizumab for women with newly diagnosed ovarian cancer (ICON7): Overall survival results of a phase 3 randomised trial. Lancet Oncol 16: 928-936, 2015.

5. Burger RA, Brady MF, Bookman MA, Fleming GF, Monk BJ, Huang H, Mannel RS, Homesley HD, Fowler J, Greer BE, et al: Incorporation of bevacizumab in the primary treatment of ovarian cancer. N Engl J Med 365: 2473-2483, 2011.

6. Aghajanian C, Blank SV, Goff BA, Judson PL, Teneriello MG, Husain A, Sovak MA, Yi J and Nycum LR: OCEANS: A randomized, double-blind, placebo-controlled phase III trial of chemotherapy with or without bevacizumab in patients with platinum-sensitive recurrent epithelial ovarian, primary peritoneal, or fallopian tube cancer. J Clin Oncol 30: 2039-2045, 2012.

7. Pujade-Lauraine E, Hilpert F, Weber B, Reuss A, Poveda A, Kristensen G, Sorio R, Vergote I, Witteveen P, Bamias A, et al Bevacizumab combined with chemotherapy for platinum resistant recurrent ovarian cancer: The AURELIA open-label randomized phase III trial. J Clin Oncol 32: 1302-1308, 2014.

8. du Bois A, Floquet A, Kim JW, Rau J, del Campo JM, Friedlander M, Pignata S, Fujiwara K, Vergote I, Colombo N, et al Incorporation of pazopanib in maintenance therapy of ovarian cancer. J Clin Oncol 32: 3374-3382, 2014.

9. du Bois A, Kristensen G, Ray-Coquard I, Reuss A, Pignata S, Colombo N, Denison U, Vergote I, Del Campo JM, Ottevanger $\mathrm{P}$, et al: Standard first-line chemotherapy with or without nintedanib for advanced ovarian cancer (AGO-OVAR 12): A randomised, double blind, placebo-controlled phase 3 trial. Lancet Oncol 17: 78-89, 2016.

10. Wang X, Shen Y, Li S, Lv M, Zhang X, Yang J, Wang F and Yang J: Importance of the interaction between immune cells and tumor vasculature mediated by thalidomide in cancer treatment (Review). Int J Mol Med 38: 1021-1029, 2016.

11. Sherbet GV: Therapeutic potential of thalidomide and its analogues in the treatment of cancer. Anticancer Res 35: 5767-5772, 2015

12. Bauer JA, Morrison BH, Grane RW, Jacobs BS, Borden EC and Lindner DJ: IFN-alpha2b and thalidomide synergistically inhibit tumor-induced angiogenesis. J Interferon Cytokine Res 23: 3-10, 2003.

13. Vargesson N: Thalidomide-induced limb defects: Resolving a 50-year-old puzzle. Bioessays 31: 1327-1336, 2009.

14. McBride W: Thalidomide and congenital malformations. Lancet 1: 358, 1961

15. Connors T: Anticancer drug development: The way forward. Oncologist 1: 180-181, 1996.

16. Bramuzzo M, Ventura A, Martelossi S and Lazzerini M: Thalidomide for inflammatory bowel disease: Systematic review. Medicine (Baltimore) 95: e4239, 2016.

17. Milanovic D, Sticht C, Röhrich M, Maier P, Grosu AL and Herskind C: Inhibition of 13-cis retinoic acid-induced gene expression of reactive-resistance genes by thalidomide in glioblastoma tumours in vivo. Oncotarget 6: 28938-28948, 2015.

18. Liang PC, Ch'ang HJ, Hsu C, Chen LT, Shih TT and Liu TW: Perfusion parameters of dynamic contrast-enhanced magnetic resonance imaging predict outcomes of hepatocellular carcinoma receiving radiotherapy with or without thalidomide. Hepatol Int 9: 258-268, 2015 .

19. Gao M, Kong Y, Wang H, Xie B, Yang G, Gao L, Zhang Y, Zhan F, Dai B, Tao Y and Shi J: Thalidomide treatment for patients with previously untreated multiple myeloma: A meta-analysis of randomized controlled trials. Tumour Biol 37: 11081-11098, 2016.

20. Guirguis AA and Ebert BL: Lenalidomide: Deciphering mechanisms of action in myeloma, myelodysplastic syndrome and beyond. Curr Opin Cell Biol 37: 61-67, 2015.

21. Galustian C and Dalgleish A: Lenalidomide: A novel anticancer drug with multiple modalities. Expert Opin Pharmacother 10: 125-133, 2009.

22. Kanwar VS, Heath J, Krasner CN and Pearce JM: Advanced small cell carcinoma of the ovary in a seventeen-year-old female, successfully treated with surgery and multi-agent chemotherapy. Pediatr Blood Cancer 50: 1060-1062, 2008.

23. Phippen NT and Leath CA III: Weekly topotecan and daily thalidomide in patients with recurrent epithelial ovarian cancer: A report of 2 cases. J Reprod Med 54: 583-586, 2009.
24. Buttin BM and Moore MJ: Thalidomide-induced reversible interstitial pneumonitis in a patient with recurrent ovarian cancer. Gynecol Oncol 111: 546-548, 2008.

25. Braun AG and Weinreb SL: Teratogen metabolism: Spontaneous decay products of thalidomide and thalidomide analogues are not bioactivated by liver microsomes. Teratog Carcinog Mutagen 5: 149-158, 1985.

26. Braun AG and Dailey JP: Thalidomide metabolite inhibits tumor cell attachment to concanavalin A coated surfaces. Biochem Biophys Res Commun 98: 1029-1034, 1981.

27. Bauer JA, Morrison BH, Grane RW, Jacobs BS, Borden EC and Lindner DJ: IFN- alpha2b and thalidomide synergistically inhibit tumor-induced angiogenesis. J Interferon Cytokine Res 23: 3-10, 2003.

28. Kobayashi H, Yagyu T, Kondo T, Kurita N, Inagaki K, Haruta S, Kawaguchi R, Kitanaka T, Sakamoto Y, Yamada Y, et al: Suppression of urokinase receptor expression by thalidomide is associated with inhibition of nuclear factor kappaB activation and subsequently suppressed ovarian cancer dissemination. Cancer Res 65: 10464-10471, 2005.

29. Li W, Peng ZL and Cao ZY: Study of thalidomide on the growth and angiogenesis of ovary cancer SKOV3 transplanted subcutaneously in nude mice. Zhonghua Fu Chan Ke Za Zhi 40: 186-189, 2005 (In Chinese)

30. Strese S, Fryknäs M, Larsson R and Gullbo J: Effects of hypoxia on human cancer cell line chemosensitivity. BMC Cancer 13: 331, 2013.

31. Piura B, Medina L, Rabinovich A, Dyomin V and Huleihel M: Thalidomide distinctly affected TNF-8.5, IL-6 and MMP secretion by an ovarian cancer cell line (SKOV-3) and primary ovarian cancer cells. Eur Cytokine Netw 24: 122-129, 2013.

32. Eisen TG: Thalidomide in solid tumors: The London experience. Oncology (Williston Park) 14 (12 Suppl 13): S17-S20, 2000.

33. Eleutherakis-Papaiakovou V, Bamias A and Dimopoulos MA: Thalidomide in cancer medicine. Ann Oncol 15: 1151-1160, 2004.

34. Ma WW and Jimeno A: Strategies for suppressing angiogenesis in gynecological cancers. Drugs Today (Barc) 43: 259-273, 2007.

35. Galustian C and Dalgleish A: Lenalidomide: A novel anticancer drug with multiple modalities. Expert Opin Pharmacother 10: 125-133, 2009.

36. Malayev Y, Levene R and Gonzalez F: Palliative chemotherapy for malignant ascites secondary to ovarian cancer. Am J Hosp Palliat Care 29: 515-521, 2012.

37. Gadducci A, Sergiampietri C and Guiggi I: Antiangiogenic agents in advanced, persistent or recurrent endometrial cancer: A novel treatment option. Gynecol Endocrinol 29: 811-816, 2013.

38. Benesch M, Lackner H, Pilhatsch A, Gürtl-Lackner B, Schwinger $\mathrm{W}$ and Urban C: Long-term remission in a female with multiple relapsed juvenile granulosa cell tumor. J Pediatr Hematol Oncol 37: e486-e489, 2015.

39. Jeyakumar A, Chalas E and Hindenburg A: Sustained complete remission in a patient with platinum-resistant ovarian yolk sac tumor. Gynecol Oncol 82: 578-580, 2001

40. Eisen T, Boshoff C, Mak I, Sapunar F, Vaughan MM, Pyle L, Johnston SR, Ahern R, Smith IE and Gore ME: Continuous low dose Thalidomide: A phase II study in advanced melanoma, renal cell, ovarian and breast cancer. Br J Cancer 82: 812-817, 2000.

41. Abramson N, Stokes PK, Luke M, Marks AR and Harris JM: Ovarian and papillary- serous peritoneal carcinoma: Pilot study with thalidomide. J Clin Oncol 20: 1147-1149, 2002.

42. Gordinier ME, Dizon DS, Weitzen S, Disilvestro PA, Moore RG and Granai CO: Oral thalidomide as palliative chemotherapy in women with advanced ovarian cancer. J Palliat Med 10: 61-66, 2007.

43. Zhang MM, Chan JK, Husain A, Guo HY and Teng NN: Safety and efficacy of lenalidomide (Revlimid) in recurrent ovarian and primary peritoneal carcinoma. Gynecol Oncol 105: 194-198, 2007.

44. Chan JK, Manuel MR, Ciaravino G, Cheung MK, Husain A and Teng NN: Safety and efficacy of thalidomide in recurrent epithelial ovarian and peritoneal carcinoma. Gynecol Oncol 103: 919-923, 2006

45. Downs LS Jr, Judson PL, Argenta PA, Ghebre R, Geller MA, Bliss RL, Boente MP, Nahhas WA, Abu-Ghazaleh SZ, Chen MD and Carson LF: A prospective randomized trial of thalidomide with topotecan compared with topotecan alone in women with recurrent epithelial ovarian carcinoma. Cancer 112: 331-339, 2008. 
46. Hurteau JA, Brady MF, Darcy KM, McGuire WP, Edmonds P, Pearl ML, Ivanov I, Tewari KS, Mannel RS, Zanotti K and Benbrook DM: Randomized phase III trial of tamoxifen versus thalidomide in women with biochemical-recurrent-only epithelial ovarian, fallopian tube or primary peritoneal carcinoma after a complete response to first-line platinum/taxane chemotherapy with an evaluation of serum vascular endothelial growth factor (VEGF): A gynecologic oncology group study. Gynecol Oncol 119: 444-450, 2010.

47. Carter JS and Downs LS Jr: A prospective clinical trial of lenalidomide with topotecan in women with advanced epithelial ovarian carcinoma. Int J Clin Oncol 16: 666-670, 2011.

48. Muthuramalingam SR, Braybrooke JP, Blann AD, Madhusudan S, Wilner S, Jenkins A, Han C, Kaur K, Perren T and Ganesan TS: A prospective randomised phase II trial of thalidomide with carboplatin compared with carboplatin alone as a first-line therapy in women with ovarian cancer, with evaluation of potential surrogate markers of angiogenesis. Eur J Gynaecol Oncol 32: 253-258, 2011.

49. Ganesan P, Piha-Paul S, Naing A, Falchook G, Wheler J, Fu S, Hong DS, Kurzrock R, Janku F, Laday S, et al: Phase I clinical trial of lenalidomide in combination with sorafenib in patients with advanced cancer. Invest New Drugs 32: 279-286, 2014.

50. SelleF,SevinE, Ray-CoquardI,Mari V,Berton-RigaudD,FavierL, Fabbro M, Lesoin A, Lortholary A and Pujade-Lauraine E: A phase II study of lenalidomide in platinum-sensitive recurrent ovarian carcinoma. Ann Oncol 25: 2191-2196, 2014.
51. Tilluckdharry L, Dean R, Farver C and Ahmad M: Thalidomide-related eosinophilic pneumonia: A case report and brief literature review. Cases J 1: 143, 2008

52. Gordinier ME and Dizon DS: Dyspnea during thalidomide treatment for advanced ovarian cancer. Ann Pharmacother 39: 962-965, 2005.

53. Santi RM, Ceccarelli M, Catania G, Monagheddu C, Evangelista A, Bernocco E, Monaco F, Federico M, Vitolo U, Cortelazzo S, et al: PO-03 - Khorana score and histotype predict the incidence of early venous thromboembolism (VTE) in non hodgkin lymphoma (NHL). A pooled data analysis of twelve clinical trials of fondazione italiana linfomi (FIL). Thromb Res 140 (Suppl 1): S177, 2016.

54. Joerger M, Schaer-Thuer C, Koeberle D, Matter-Walstra K, Gibbons-Marsico J, Diem S, Thuerlimann B and Cerny T: Off-label use of anticancer drugs in eastern Switzerland: A population-based prospective cohort study. Eur J Clin Pharmacol 70: 719-725, 2014.

55. Dasanu CA, Mewawalla P and Grabska J: Multiple myeloma and its therapies: to what extent do they contribute to the increased incidence of second malignant neoplasms? Curr Med Res Opin 28: 1129-1140, 2012. 